Check for updates

Cite this: RSC Adv., 2019, 9, 14718

Received 24th April 2019

Accepted 6th May 2019

DOI: $10.1039 / c 9 r a 03042 e$

rsc.li/rsc-advances

\section{Electronic and optical absorption properties of organic-inorganic perovskites as influenced by different long-chain diamine molecules: first- principles calculations $\uparrow$}

\author{
Wangzhe Zhou, Dan Li, (DD * Di Zhang, Hu Tang, He Zhang and Chunjun Liang (DD *
}

Organic-inorganic perovskites have demonstrated significant promise as photovoltaic materials due to their excellent photoelectric properties. However, monoamino three-dimensional (3D) perovskites, such as $\mathrm{CH}_{3} \mathrm{NH}_{3} \mathrm{Pbl}_{3}\left(\mathrm{MAPb}_{3}\right)$ and $\mathrm{NH}_{2} \mathrm{CHNH}_{2} \mathrm{Pbl}_{3}\left(\mathrm{FAPbl}_{3}\right)$ exhibit low thermal and chemical stability, leading to low device durability. As such, we sought to address this problem by evaluating the performance of five diamino$3 \mathrm{D}$ perovskites with different molecule chain lengths, including $\mathrm{NH}_{3}\left(\mathrm{CH}_{2}\right)_{2} \mathrm{NH}_{3} \mathrm{Pbl}_{4}$ (EDAPbl$)_{4}$, $\mathrm{NH}_{3}\left(\mathrm{CH}_{2}\right)_{3} \mathrm{NH}_{3} \mathrm{Pbl}_{4} \quad\left(\mathrm{DPAPbl}_{4}\right), \quad \mathrm{NH}_{3}\left(\mathrm{CH}_{2}\right)_{4} \mathrm{NH}_{3} \mathrm{Pbl}_{4} \quad\left(\mathrm{BDAPbl}_{4}\right), \quad \mathrm{NH}_{3}\left(\mathrm{CH}_{2}\right)_{5} \mathrm{NH}_{3} \mathrm{Pbl}_{4} \quad\left(\mathrm{PDAPbl}_{4}\right), \quad$ and $\mathrm{NH}_{3}\left(\mathrm{CH}_{2}\right)_{6} \mathrm{NH}_{3} \mathrm{Pbl}_{4}\left(\mathrm{HDAPbl}_{4}\right)$, as well as one monoamino-2D perovskite, $\left(\mathrm{CH}_{3}\left(\mathrm{CH}_{2}\right)_{3} \mathrm{NH}_{3}\right)_{2} \mathrm{Pbl}_{4}\left(\mathrm{BA}_{2} \mathrm{Pbl}_{4}\right)$ using first-principles calculations. We analyzed the geometries, formation energies, electronic structures, and optical absorption properties of each of these materials. We determined the composition of the conduction and valence bands and analyzed the charge transfer between the inorganic layer and organic molecules. The transport characteristics of the electrons in the different directions were analyzed by calculating the effective mass in different directions. Based on these results, $\mathrm{BDAPbl}_{4}$ was predicted to exhibit the best photovoltaic performance, as well as demonstrating a light effective mass of the electrons and holes, a reduced bandgap, and a large optical absorption, compared to the other perovskites assessed in this study.

\section{Introduction}

Since the development of organic-inorganic halide perovskite solar cells in 2009, ${ }^{1}$ their importance in the field of solar power research has increased substantially. After several years of development, these solar cells have demonstrated many advantages, including an appropriate bandgap, high absorption coefficient, high carrier mobility, and low carrier recombination rate. $^{2}$ The power conversion efficiency of $3 \mathrm{D}$ perovskites has rapidly reached $23.7 \%,{ }^{3}$ showing great commercial potential. However, 3D perovskites have issues with low luminescence activity and low stability regarding heat and moisture., ${ }^{\mathbf{4} 5}$ Generally, the structure of 3D perovskites consists of $\mathrm{PbX}_{3}(\mathrm{X}=$ $\mathrm{Cl}, \mathrm{Br}$, and I) as the skeleton and methylamine (MA) at the center of the structure. With this structure, internal factors such as ion migration, electron transfer, and interfacial reaction ${ }^{6-8}$ as well as external factors such as humidity and oxidant ${ }^{9,10}$ can have important effects on its stability. For example, in Schoonman et al., the $\mathrm{Pb}-\mathrm{I}$ bond component in organic-inorganic hybridized methylammonium lead-halide perovskite can

Department of Physics, Beijing Jiaotong University, Beijing 1001044, People's Republic of China.E-mail: danli@bjtu.edu.cn; chjliang@bjtu.edu.cn

$\dagger$ Electronic supplementary information (ESI) available. See DOI: 10.1039/c9ra03042e lead to photolysis. ${ }^{\mathbf{1 1}}$ Therefore, researchers have introduced the use of $2 \mathrm{D}$ perovskites to solve such problems. ${ }^{12-15}$ The existence of the organic layer enhances the stability of the compound to heat and moisture, ${ }^{16-21}$ but it also leads to a high bandgap, with the bandgap for $\left(\mathrm{CH}_{3}\left(\mathrm{CH}_{2}\right)_{3} \mathrm{NH}_{3}\right)_{2} \mathrm{PbI}_{4}$ and $\left(\mathrm{CH}_{3}\left(\mathrm{CH}_{2}\right)_{3} \mathrm{NH}_{3}\right)_{2}(-$ $\left.\mathrm{CH}_{3} \mathrm{NH}_{3}\right) \mathrm{Pb}_{2} \mathrm{I}_{7}$ calculated by Stoumpos et al. being $2.43 \mathrm{eV}$ and $2.17 \mathrm{eV}$, respectively, ${ }^{17}$ less optical absorption, low interlayer mobility of carriers, ${ }^{\mathbf{1 8 , 1 9}}$ and reduced energy conversion efficiency, ${ }^{\mathbf{1 9} 20}$ which limits the application of $2 \mathrm{D}$ perovskite materials in photovoltaic harvesters.

In this paper, we use diamino groups as organic substances to form 3D perovskites. Considering the instability of shortchain molecules, such as methylamine (MA) and formamidine (FA), we have introduced long-chain molecules, including 1,3diaminopropane (DPA), 1,4-butanediamine (BDA), and 1,5pentanediamine (PDA). We investigated whether these diamino-3D perovskites had increased stability, in an effort to address the instability problem of organic-inorganic halide perovskites, and if they could maintain the excellent characteristics of perovskites, such as their low loss, high carrier mobility, and large optical absorption. In order to compare the diamino-3D perovskites, we also produced a $2 \mathrm{D}$ perovskite whose organic molecules consisted of monoamino butylamine (BA), with the amino groups of the two BA molecules attracted to the inorganic layer and the other end serving as a vacuum 
layer. We studied the geometries, formation energies, electronic structures, and optical absorption properties of monoamino-2D perovskite $\left(\mathrm{BA}_{2} \mathrm{PbI}_{4}\right)$ and the diamino-3D perovskites (EDAPbI $\mathrm{DPAPbI}_{4}, \mathrm{BDAPbI}_{4}, \mathrm{PDAPbI}_{4}$, and $\mathrm{HDAPbI}_{4}$ ) using the firstprinciples method based on density functional theory (DFT). This study will help in the design and synthesis of more efficient and stable organic-inorganic perovskite optoelectronic devices.

\section{Computational methodology}

The first-principles calculations were performed using the Vienna Ab initio Simulation Package (VASP), ${ }^{22}$ the electron-ion interaction was described using the projection-enhanced plane wave (PAW) pseudopotential, ${ }^{23}$ and the Perdew-Burke-Ernzerhof (PBE) of generalized gradient approximation (GGA) was used to describe the exchange-correlation energy between the electrons. ${ }^{24}$ Electronic orbitals 5d6s6p, 5s5p, 2s2p, 2s2p, and 1s were considered in valence for $\mathrm{Pb}, \mathrm{I}, \mathrm{C}, \mathrm{N}$, and $\mathrm{H}$ atoms, respectively. The cut-off energy for the plane wave basis set was $500 \mathrm{eV}$. The convergence criterion of the self-consistent calculations for ionic relaxations was $10^{-5} \mathrm{eV}$ between two consecutive steps. The first Brillouin zone was sampled using a gammacentered method to generate $k$-point meshes. In the structural optimization, the K-point grid size for $\mathrm{BA}_{2} \mathrm{PbI}_{4}$ was $4 \times 4 \times 1$, as it had a vacuum layer in the $c$ direction; the K-point grid size for diamino-3D perovskites was $4 \times 4 \times 4$. In order to acquire a more accurate wave function and charge density, $16 \times 16 \times 1$ and $16 \times 16 \times 16 \mathrm{k}$-point grids were used in the static calculation. Considering the influence of intermolecular forces on the calculation results, a van der Waals correction was performed via the zero damping DFT-D3 method of Grimme in VASP. ${ }^{25}$ The charge densities of the VBM states and CBM states on each atom were integrated using Bader analysis. ${ }^{26-28}$

The band structure calculation of $\mathrm{MAPbI}_{3}$ using the $\mathrm{PBE}$ exchange-correlation functional of GGA shows the well-known weakness of band gap underestimation is not found, GGA band gaps are very similar with the experimental values. ${ }^{29}$ It is generally believed that this accidental agreement is from the error cancelation, i.e., neglecting the spin-orbit interaction caused band gap overestimation, which cancels the underestimation error of GGA calculation..$^{30}$ The strong SOC effect results in a very large splitting of the CBM states, the underestimated band gap is corrected by HSE06 functional or GW self-energy approach incorporating SOC. For example, the band gaps of $\mathrm{BA}_{2} \mathrm{PbI}_{4}$ and $\mathrm{MAPbI}_{3}$ calculated by Biao Liu et al. using HSE + SOC functional calculation are about $0.1 \mathrm{eV}$ lower than PBE. ${ }^{31}$ SOC-GW delivers calculated band gaps $1.67 \mathrm{eV}$ for $\mathrm{MAPbI}_{3}$ in excellent agreement with the experimental values. ${ }^{32}$ But SOC has a very small effect on the atomic structure and ground-state properties. ${ }^{33}$ Therefore, we adopt the PBE exchange-correlation as a practical choice to reflect the real band gap.

\section{Results and discussion}

\subsection{Geometric structure and formation energy}

The optimized structures of $\mathrm{BA}_{2} \mathrm{PbI}_{4}, \mathrm{DPAPbI}_{4}, \mathrm{BDA}_{2} \mathrm{PbI}_{4}$, and $\mathrm{PDAPbI}_{4}$ with minimum energy after ion relaxation are shown in Fig. 1. The optimized structures of EDAPbI 4 and $\mathrm{HDAPbI}_{4}$ are shown in Fig. S1. $\dagger$ The amino group (BA) is positively charged and adsorbs with the negatively charged $\mathrm{Pb}-\mathrm{I}$ inorganic layer. Therefore, we use a structure in which two single amino groups (BA) are adsorbed by an inorganic layer to form 2D perovskite $\left(\mathrm{BA}_{2} \mathrm{PbI}_{4}\right)$, and a structure in which a double amino group and upper and lower inorganic layers are used to form 3D perovskites $\left(\mathrm{EDAPbI}_{4}, \mathrm{DPAPbI}_{4}, \mathrm{BDAPbI}_{4}, \mathrm{PDAPbI}_{4}\right.$, and $\left.\mathrm{HDAPbI}_{4}\right)$. As shown in Fig. 1(c) and (d), it can be seen that with the increased length of the main chain of the diamino molecule in the 3D perovskite, the molecular chain is bent, which may have been

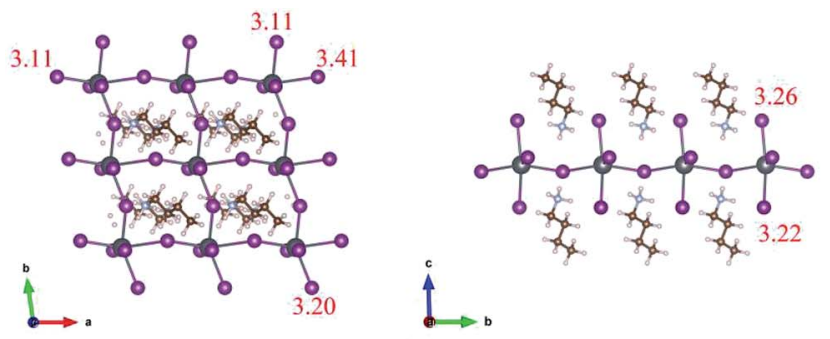

(a)
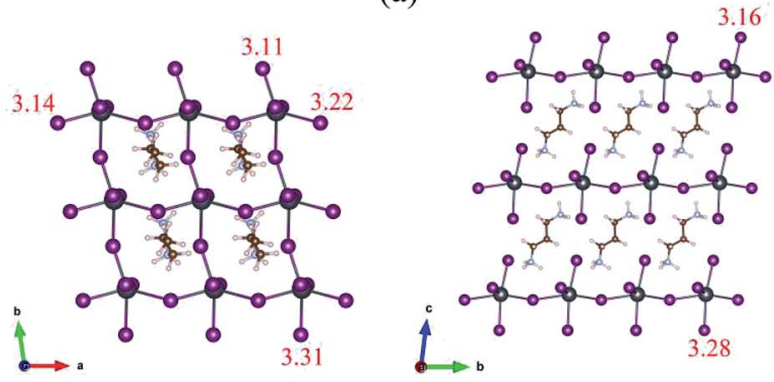

(b)
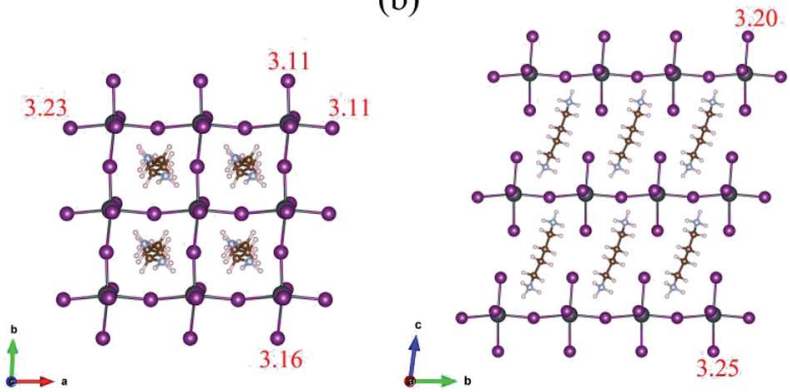

(c)
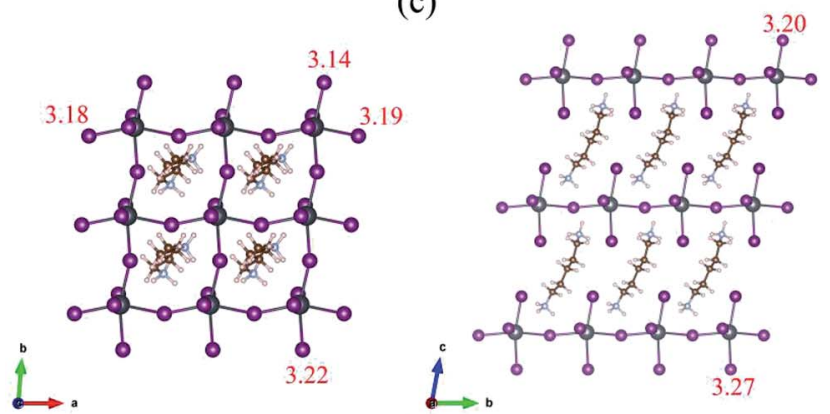

(d)

Fig. 1 The optimized geometrical structures of (a) $\mathrm{BA}_{2} \mathrm{Pbl}_{4}$, (b) $\mathrm{DPAPbl}_{4}$, (c) $\mathrm{BDAPbl}_{4}$, and (d) $\mathrm{PDAPbl}_{4}$ perovskites. The purple, gray, brown, silver, and smallest balls represent $\mathrm{I}, \mathrm{Pb}, \mathrm{C}, \mathrm{N}$, and $\mathrm{H}$ atoms, respectively. The red number is the length of the corresponding $\mathrm{Pb}-\mathrm{I}$ bond in angstroms. 
caused by the soft skeleton of the long chain molecule. Because long-chain diamino molecules interact with the upper and lower inorganic layers, the supercell deviates from the orthogonal symmetry and degenerates into triclinic symmetry. The degree of supercell tilt is characterized by the value of $|\Delta \alpha|+$ $|\Delta \beta|+|\Delta \gamma|$. Here, $\Delta \alpha, \Delta \beta$, and $\Delta \gamma$ are the differences in the angle between the base vectors and the 90 degree angle, respectively. From the data presented in Table 1 , among the five kinds of diamino-3D perovskites, the values of $|\Delta \alpha|+|\Delta \beta|+$ $|\Delta \gamma|$ for $\mathrm{EDAPbI}_{4}, \mathrm{BDAPbI}_{4}$, and $\mathrm{HDAPbI}_{4}$ are relatively small, at $6.9,13.9$, and 15.3 degrees, respectively. However, the values of $|\Delta \alpha|+|\Delta \beta|+|\Delta \gamma|$ for $\mathrm{DPAPbI}_{4}$ and $\mathrm{PDAPbI}_{4}$ are 17.3 and 24.6 degrees, respectively, which are relatively large. It was found that the perovskite structure had a higher symmetry when the number of atoms in the main chain of the diamino molecules was even. For example, in $\mathrm{EDAPbI}_{4}, \mathrm{BDAPbI}_{4}$, and $\mathrm{HDAPbI}_{4}$ there are two $\mathrm{N}$ atoms and two, four, and six $\mathrm{C}$ atoms in the main chain of the diamino molecule, respectively. When the number of atoms in the main chain was even, the molecule had an inversion symmetry. It was observed that the inversion symmetry molecules in the inorganic skeleton generally did not rotate along the axis, but they only bent with the increase of the length of the molecular chain, as shown in Fig. 1(c), S1(a) and (b). $\uparrow$ It was shown that the deformation of the inorganic skeleton formed by $\mathrm{Pb}$ and I was relatively small, leading to a smaller deviation from the orthogonal symmetry. In contrast, when the number of atoms in the main chain was odd, the molecules did not demonstrate inversion symmetry but had a mirror symmetry instead, such as $\mathrm{DPAPbI}_{4}$ and $\mathrm{PDAPbI}_{4}$, which have two $\mathrm{N}$ atoms as well as three and five $\mathrm{C}$ atoms in the main chain of the diamino molecule, respectively. It was found that the molecules without inversion symmetry rotate along the axis in order to maintain the inversion symmetry of the inorganic skeleton; correspondingly, the inorganic $\mathrm{Pb}-\mathrm{I}$ layers are also obviously distorted, as shown in Fig. 1(b) and (d). In addition, it is important to note that the bandgap and band structure depends on the symmetry of the crystal, which is discussed below. Obviously, the lattice constant $c$ increased with the increase in molecular chain length, from the lattice constant $c$ of $9.40 \AA$ for $\mathrm{EDAPbI}_{4}$ to that of $12.91 \AA$ for $\mathrm{HDAPbI}_{4}$ (Table 1). In (BA) ${ }_{2} \mathrm{PbI}_{4}$, a monolayer $2 \mathrm{D}$ perovskite was formed by monoamino molecular ions as organic layers. Because two long chain monoamino ions (BA) interact with only one inorganic $\mathrm{Pb}-\mathrm{I}_{4}$ layer, the geometric structure of the atoms in the plane of the $\mathrm{Pb}-\mathrm{I}_{4}$ layer was greatly affected, resulting in a larger deviation of the $\gamma$ angle from a right angle, while the $\alpha$ and $\beta$ angles were basically right angles (Table 1).

The following formulas were used to calculate the formation energy $\left(E_{\mathrm{f}}\right)$ of the monoamine-2D perovskite and diamino-3D perovskites:

$$
\begin{gathered}
E_{\mathrm{f}}\left[(\mathrm{BA})_{2} \mathrm{PbI}_{4}\right]=E\left[(\mathrm{BA})_{2} \mathrm{PbI}_{4}\right]-E\left(\mathrm{PbI}_{2}\right)-2 E(\mathrm{BAI}) \\
E_{\mathrm{f}}\left[\mathrm{DPbI}_{4}\right]=E\left[\mathrm{DPbI}_{4}\right]-E\left(\mathrm{PbI}_{2}\right)-E\left[\mathrm{DI}_{2}\right]
\end{gathered}
$$

where D denotes diamino molecules EDA, DPA, BDA, PDA, and HDA. The $E\left[(\mathrm{BA})_{2} \mathrm{PbI}_{4}\right]$ and $E\left[\mathrm{DPbI}_{4}\right]$ are the DFT total energies for the $2 \mathrm{D}$ perovskite $\left(\mathrm{BA}_{2} \mathrm{PbI}_{4}\right)$ and $3 \mathrm{D}$ perovskites (EDAPbI 4 , $\mathrm{DPAPbI}_{4}, \mathrm{BDAPbI}_{4}, \mathrm{PDAPbI}_{4}$, and $\mathrm{HDAPbI}_{4}$ ), which were synthesized from crystalline $\mathrm{BAI}, \mathrm{DI}_{2}$, and $\mathrm{PbI}_{2}$, respectively. The $E(\mathrm{BAI}), E\left[\mathrm{DI}_{2}\right]$, and $E\left[\mathrm{PbI}_{2}\right]$ are the DFT total energies of each reactant.

The calculated formation energies of the $2 \mathrm{D}$ perovskite $\left(\mathrm{BA}_{2} \mathrm{PbI}_{4}\right)$ and $3 \mathrm{D}$ perovskites $\left(\mathrm{EDAPbI}_{4}, \mathrm{DPAPbI}_{4}, \mathrm{BDAPbI}_{4}\right.$, $\mathrm{PDAPbI}_{4}$, and $\mathrm{HDAPbI}_{4}$ ) were $0.37,0.49,0.26,0.29,-0.01$, and $0.05 \mathrm{eV}$, respectively (see Table 1 ). The positive formation energies indicated that the synthesis reaction was an endothermic reaction, and the negative formation energies show that the reactions were exothermic. There is no doubt that $\mathrm{PDAPbI}_{4}$ is the easiest to synthesize. In general, the formation energy of perovskite materials formed by diamino molecules with longer main chains was relatively low (Table 1). The reason may be that the skeleton of long chain molecules is softer and easier to deform, resulting in lower formation energy. In addition, because the $\mathrm{Pb}-\mathrm{I}$ inorganic layer is negatively charged, electrostatic repulsion occurs between two adjacent layers. When the length of the chain becomes longer, the inorganic layer spacing increases. The electrostatic attraction energy between the amino group and the inorganic layer remained basically unchanged, and the reduction of the electrostatic repulsion energy also resulted in the reduction of the formation energy. Moreover, the formation energy of the diamino-3D $\mathrm{DPAPbI}_{4}$ perovskite was lower than that of the monoamino-2D $\mathrm{BA}_{2} \mathrm{PbI}_{4}$ perovskite, although they have the same main chain length. In order to compare with the formation energy of traditional perovskite $\left(\mathrm{MAPbI}_{3}\right.$ and $\left.\mathrm{FAPbI}_{3}\right)$, we also calculated the formation energy of per primitive for pseudo-cubic $\mathrm{MAPbI}_{3}$ and $\mathrm{FAPbI}_{3}$, which are 0.08 and $-0.04 \mathrm{eV}$, respectively, close to the formation energies of $\mathrm{PDAPbI}_{4}$ and $\mathrm{HDAPbI}_{4}$ with long chain diamino molecules. Although the negative and

Table 1 Lattice parameters $(a, b, c)$, the distance between I atoms in the two adjacent inorganic layers in the $c$ direction $(I-I)$, the calculated

\begin{tabular}{|c|c|c|c|c|c|c|c|c|c|c|}
\hline Compounds & $a$ & $b$ & $c$ & $\alpha$ & $\beta$ & $\gamma$ & $|\Delta \alpha|+|\Delta \beta|+|\Delta \gamma|$ & $\mathrm{I}-\mathrm{I}$ & $E_{\mathrm{f}}$ & $E_{\mathrm{g}}$ \\
\hline$(\mathrm{BA})_{2} \mathrm{PbI}_{4}$ & 6.35 & 6.06 & - & 91.6 & 89.4 & 98.1 & 10.3 & - & 0.37 & 2.49 \\
\hline $\mathrm{EDAPbI}_{4}$ & 6.42 & 6.04 & 9.40 & 89.6 & 96.3 & 90.2 & 6.9 & 3.97 & 0.49 & 2.30 \\
\hline $\mathrm{DPAPbI}_{4}$ & 6.06 & 6.25 & 10.20 & 83.5 & 87.0 & 97.7 & 17.3 & 3.83 & 0.26 & 2.22 \\
\hline $\mathrm{BDAPbI}_{4}$ & 6.29 & 6.23 & 10.61 & 82.7 & 94.9 & 88.3 & 13.9 & 4.25 & 0.29 & 1.78 \\
\hline $\mathrm{PDAPbI}_{4}$ & 6.24 & 6.28 & 11.52 & 78.9 & 82.5 & 84.1 & 24.6 & 5.21 & -0.01 & 2.18 \\
\hline $\mathrm{HDAPbI}_{4}$ & 6.36 & 6.12 & 12.91 & 89.7 & 103.7 & 91.3 & 15.3 & 6.42 & 0.05 & 2.03 \\
\hline
\end{tabular}
formation energy $\left(E_{\mathrm{f}}\right)$, and bandgap $\left(E_{\mathrm{g}}\right)$. Lattice lengths and "I-I" given in $\AA$, lattice angles are given in ${ }^{\circ}, E_{\mathrm{f}}$ and $E_{\mathrm{g}}$ given in eV 
significantly low formation energies indicate that $\mathrm{PDAPbI}_{4}$ and $\mathrm{HDAPbI}_{4}$ are easier synthesize in experiments, appropriate large formation energy can enhance the stability of $2 \mathrm{D}$ and $3 \mathrm{D}$ perovskite. ${ }^{34}$

\subsection{Electronic properties}

The calculated energy band structures of $\mathrm{BA}_{2} \mathrm{PbI}_{4}, \mathrm{DPAPbI}_{4}$, $\mathrm{BDA}_{2} \mathrm{PbI}_{4}$, and $\mathrm{PDAPbI}_{4}$ are shown in the middle of Fig. 2. The energy band structures of $\mathrm{EDAPbI}_{4}$ and $\mathrm{HDAPbI}_{4}$ are shown in
Fig. S2. $\uparrow$ The upper left corner of Fig. 2 is the Brillouin zone of the $2 \mathrm{D}$ and $3 \mathrm{D}$ perovskites, respectively. The $2 \mathrm{D}$ material has a vacuum layer in the $c$ direction, so its Brillouin zone is also 2D. In order to take as many high symmetry points in the Brillouin zone as possible, we selected $\mathrm{G}, \mathrm{X}, \mathrm{L}_{1}, \mathrm{G}, \mathrm{Y}, \mathrm{L}_{1}, \mathrm{G}, \mathrm{L}_{2}, \mathrm{X}, \mathrm{G}$ paths and $\mathrm{N}, \mathrm{R}, \mathrm{X}, \mathrm{L}, \mathrm{R}, \mathrm{Y}, \mathrm{M}, \mathrm{R}, \mathrm{G}, \mathrm{Z}, \mathrm{R}$ paths to the perform the band calculations. We found that for $2 \mathrm{D}$ perovskite $\mathrm{BA}_{2} \mathrm{PbI}_{4}$ the valence band maximum (VBM) appeared at the $\mathrm{L}_{1}$ and $\mathrm{L}_{2}$ points, but the conduction band minimum (CBM) appeared at $\mathrm{K}$ points near $\mathrm{L}_{1}$ and $\mathrm{L}_{2}$ with a certain offset, which indicated that

Brillouin Zone

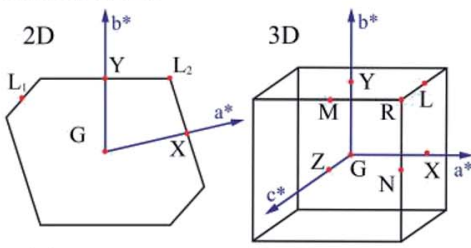

(a)

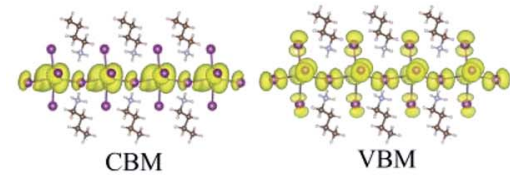

(b)

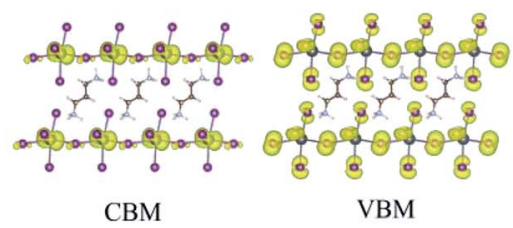

(c)

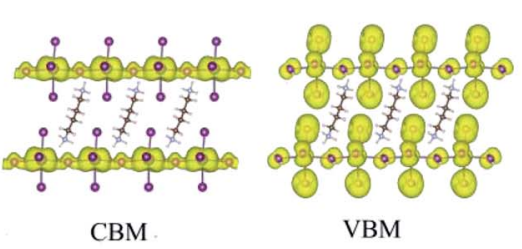

(d)

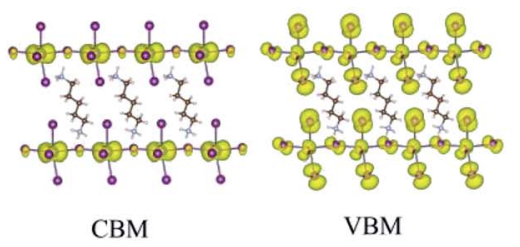

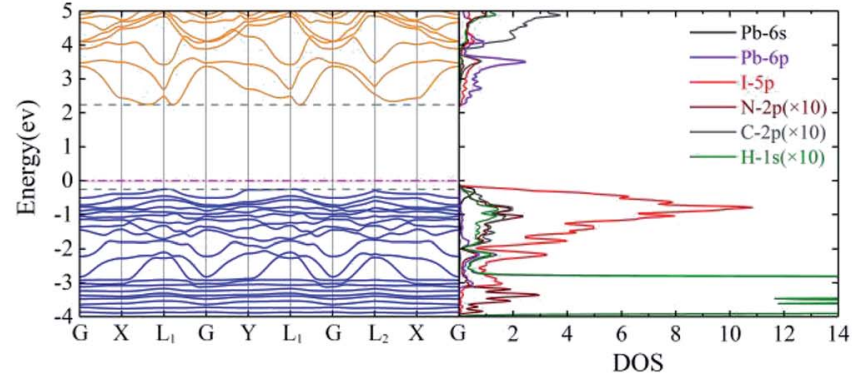
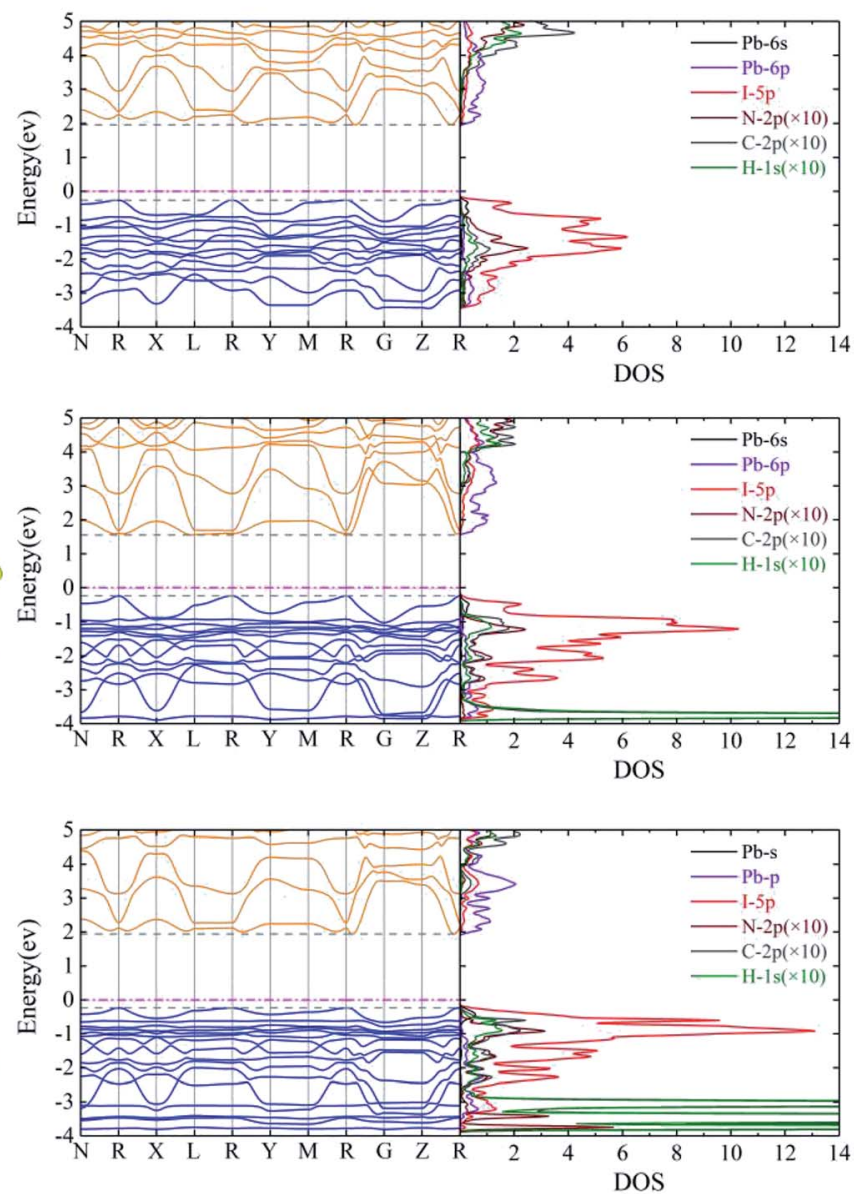

Fig. 2 From left to right, densities at the VBM and CBM states (with an isosurface value of 0.0001 e $\AA^{-3}$ ), energy band structure, and density of states (DOS) for (a) $\mathrm{BA}_{2} \mathrm{Pbl}_{4}$, (b) DPAPbl ${ }_{4}$, (c) BDAPbl 4 , and (d) PDAPbl 4 . " $\mathrm{C}-2 \mathrm{p}$ ", "N-2p", and "H-1s" are increased ten times from the original value. Fermi level is marked with a magenta line. The inset in the upper left corner indicates the high-symmetry points in the first Brillouin zone of the $2 \mathrm{D}$ and 3D perovskites. 
$\mathrm{BA}_{2} \mathrm{PbI}_{4}$ was an indirect bandgap semiconductor with a bandgap of $2.49 \mathrm{eV}$. It is interesting to note that the bandgap and band structure depended on the symmetry of the crystal. In $\mathrm{EDAPbI}_{4}, \mathrm{BDAPbI}_{4}$, and $\mathrm{HDAPbI}_{4}$ there are an even number of atoms in the main chain of the diamino molecules, which generate similar inversion symmetries, resulting in similar band dispersions near VBM and CBM, especially in the R-G and R-Y directions (Fig. 2 and $\mathrm{S} 2 \dagger$ ), and their VBM and CBM appear at the $\mathrm{R}$ point. Among the five $3 \mathrm{D}$ perovskite materials, the bandgap of $\mathrm{BDAPbI}_{4}$ was the narrowest direct bandgap at about $1.78 \mathrm{eV}$, and the band dispersion of CBM and VBM was also the largest, indicating that the effective mass of electrons and holes was relatively small, and carriers have excellent transport properties in $\mathrm{BDAPbI}_{4}$. Larger bandgaps of $2.30 \mathrm{eV}$ and $2.03 \mathrm{eV}$ were observed in the $\mathrm{EDAPbI}_{4}$ and $\mathrm{HDAPbI}_{4}$ perovskites, respectively. In contrast, in $\mathrm{DAPPbI}_{4}$ and $\mathrm{PDAPbI}_{4}$ perovskites the main chains of the diamino molecules have an odd number of atoms, and they also have similar band dispersion curves, as VBM appears at point $\mathrm{R}$ while the CBM appears near point $\mathrm{R}$ with a certain offset, indicating that they are indirect bandgap semiconductors with bandgaps of $2.22 \mathrm{eV}$ and $2.18 \mathrm{eV}$, respectively. We observed that when the molecular chain is too long (e.g. $\mathrm{HDAPbI}_{4}$ ) or too short (e.g. $\mathrm{EDAPbI}_{4}$ ), the valence band dispersion of the 3D perovskite is relatively small (Fig. S2 $\dagger$ ). For perovskite $\mathrm{EDAPbI}_{4}$, the two adjacent I atoms in the $\mathrm{C}$ direction of two adjacent inorganic layers are close to each other and repel each other, resulting in obvious position distortion of I atoms. This may result in a small overlap of the valence electron wave function. In addition, for perovskite $\mathrm{HDAPbI}_{4}$, the distance between two I atoms in the $\mathrm{C}$ direction in two adjacent inorganic layers is relatively large due to long chain molecules, which also leads to less overlap of valence band wave functions. Therefore, smaller valence band dispersion is observed in both $\mathrm{EDAPbI}_{4}$ and $\mathrm{HDAPbI}_{4}$. Overall, $\mathrm{BDAPbI}_{4}$ has the best carrier transport properties due to its suitable molecular chain length and symmetry of even molecular chains.

We also calculated the band decomposed charge density of the CBM and the VBM (left side of Fig. 2 and S2 $\dagger$ ). All 2D and 3D perovskites showed similar results, indicating that the CBM state was mainly provided by the $\mathrm{Pb}$ atoms, and the VBM state was mainly derived from the I atoms. We also observe that the I atoms contributed slightly to the CBM states; however, only the
I atoms in the $\mathrm{Pb}$ atomic layer contributed to it, and the I atoms between the two $\mathrm{Pb}$ atomic layers did not. It was shown that the effect of the six I atoms adjacent to the $\mathrm{Pb}$ atom on the formation of the energy band was not equivalent. In order to gain a deeper understanding of the electronic structures of these $2 \mathrm{D}$ and 3D perovskites, we analyzed the density of state (DOS) projected in the atomic orbital, as shown in the right side of Fig. 2 and $\mathrm{S} 2 . \dagger$ The $6 \mathrm{p}$ orbital of the $\mathrm{Pb}$ atom constituted the VBM, and the 5p orbital of I constituted the CBM. In addition, the $5 \mathrm{p}$ orbital of $\mathrm{I}$ and the $6 \mathrm{~s}$ orbital of $\mathrm{Pb}$ also contributed a little to the VBM and CBM, respectively. This is consistent with the above analysis of the band decomposed charge density of the CBM and VBM and the results of most theoretical calculations. ${ }^{35-37}$ These results indicated that the charge densities in the VBM and CBM states were distinctly separated in space. Evidently, a poor overlap of the charge density in the CBM and VBM states can lead to a low recombination transition rate in monoamino-2D and diamino-3D perovskites, which is conducive to low-loss photovoltaic devices. This result was consistent with the widely studied monoamino-3D perovskite materials $\left(\mathrm{MAPbI}_{3}, \mathrm{FAPbI}_{3}\right.$, etc.). We can see that not only the I and $\mathrm{Pb}$ atoms but also the $\mathrm{I}$ and $\mathrm{C}, \mathrm{H}$, and $\mathrm{N}$ atoms undergo orbital hybridization due to the similar DOS in the wide energy range of 2-6 eV and -3-0 eV. Therefore, the interaction between the inorganic layer and the organic molecules was mainly undertaken by the I atoms, which is consistent with the calculation results of the differential charge density presented below.

The charge density difference (Fig. 3 and S3†) is used to reflect the charge transfer between the inorganic layer and the organic molecules and is given by the following formula:

$$
\Delta \rho=\rho_{\text {perovskite }}-\rho_{\text {molecule }}-\rho_{\mathrm{PbI}_{4}}
$$

where, $\rho_{\text {perovskite }}$ and $\rho_{\text {molecule }}$ represent the charge densities of the perovskites and organic molecules after the inorganic layer is removed.

Similarly, $\rho_{\mathrm{PbI}_{4}}$ is the charge density of the inorganic $\mathrm{PbI}_{4}$ after removal of the organic molecules. The yellow and blue isosurfaces correspond to the electron increase and depletion zones, respectively. Evident charge transfers from monoamino and diamino group molecules to the I atoms were observed, as shown in Fig. 3 and S3.† The region where the electrons were

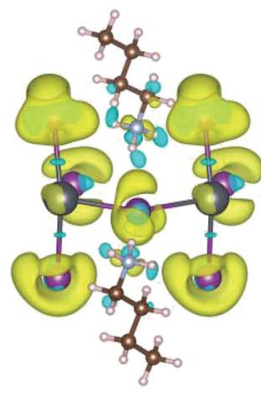

(a)

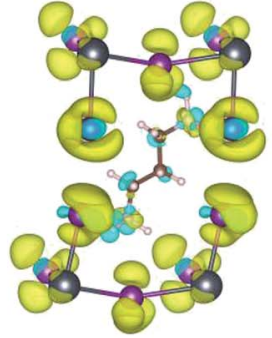

(b)

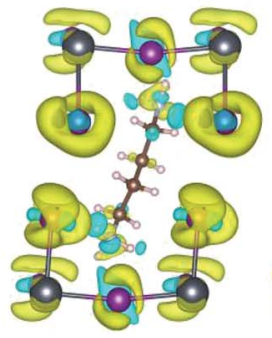

(c)

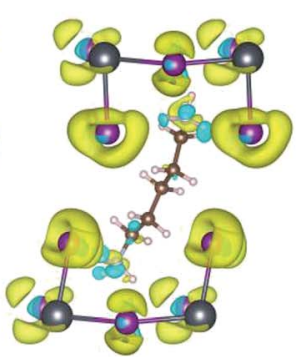

(d)

Fig. 3 Charge density difference of (a) $\mathrm{BA}_{2} \mathrm{Pbl}_{4}$, (b) $\mathrm{DPAPbl}_{4}$, (c) $\mathrm{BDAPbl}_{4}$, and (d) $\mathrm{PDAPbl}_{4}$ with an isosurface value of 0.0015 e $\AA^{-3}$. The yellow part indicates that electrons are obtained, and the blue part indicates electron loss. 
Table 2 Bader charge analysis of $\mathrm{BA}_{2} \mathrm{Pbl}_{4}, \mathrm{DPAPbl}{ }_{4}, \mathrm{BDAPbl}_{4}$, and PDAPbl ${ }_{4}$. The value in the table is in e, and the "e" represents an electron. Negative values indicate loss of electrons, and positive values indicate that electrons were obtained

\begin{tabular}{|c|c|c|c|c|c|c|c|c|c|c|c|c|}
\hline \multirow[t]{2}{*}{ Organic } & -1.4473 & $\mathrm{C}$ & -1.2086 & -1.4585 & $\mathrm{C}$ & -1.0504 & -1.4836 & $\mathrm{C}$ & -0.9316 & -1.4521 & $\mathrm{C}$ & -1.0131 \\
\hline & & $\mathrm{H}$ & -6.1407 & & $\mathrm{H}$ & -6.3297 & & $\mathrm{H}$ & -6.4050 & & $\mathrm{H}$ & -6.3304 \\
\hline \multirow[t]{2}{*}{ Inorganic } & 1.4473 & $\mathrm{~Pb}$ & -1.1080 & 1.4585 & $\mathrm{~Pb}$ & -1.0965 & 1.4836 & $\mathrm{~Pb}$ & -1.1039 & 1.4521 & $\mathrm{~Pb}$ & -1.1015 \\
\hline & & I & 2.5553 & & I & 2.5550 & & I & 2.5875 & & I & 2.5537 \\
\hline
\end{tabular}

obtained was mainly concentrated near the I atom, and the region where the monoamino and diamino groups were located lose electrons. This is also consistent with the results of our Bader charge analysis (Tables 2 and $\mathrm{S} 1 \dagger$ ). The number of electrons lost in the organic molecule of $(\mathrm{BA})_{2} \mathrm{PbI}_{4}, \mathrm{EDAPbI}_{4}$, $\mathrm{DPAPbI}_{4}, \mathrm{BDAPbI}_{4}, \mathrm{PDAPbI}_{4}$, and $\mathrm{HDAPbI}_{4}$ was about $1.45 \mathrm{e}$, $1.44 \mathrm{e}, 1.46 \mathrm{e}, 1.48 \mathrm{e}, 1.45 \mathrm{e}$, and $1.47 \mathrm{e}$, respectively. The $(\mathrm{BA})_{2} \mathrm{PbI}_{4}$ contains two monoamino molecules (BA); however, there was only one diamino molecule in the other $3 \mathrm{D}$ perovskites $\left(\mathrm{EDAPbI}_{4}, \mathrm{DPAPbI}_{4}, \mathrm{BDAPbI}_{4}, \mathrm{PDAPbI}_{4}\right.$, and $\left.\mathrm{HDAPbI}_{4}\right)$, so the number of charge losses for the diamino molecules were twice that of a single amino molecule. This shows that the monoamino molecule BA was a positive monovalent ion, while the diamino molecules (EDA, DPA, BDA, PDA, and HDA) were positive divalent ions. Therefore, it can be considered that all $2 \mathrm{D}$ and $3 \mathrm{D}$ materials were ionic, the ionicity of $\mathrm{BDAPbI}_{4}$ might be slightly stronger because the organic BDA ions lose an increased charge of about 1.48e.

We calculated the effective mass of electrons in different directions (Table 3) according to the following equation:

$$
m^{*}=\hbar^{2}\left(\frac{\partial^{2} \varepsilon(k)}{\partial k^{2}}\right)^{-1}
$$

4where $\varepsilon(k)$ are the eigenvalues at the band edges around the CBM and VBM and $k$ is the wavevector.

Table 3 Calculated electron and hole effective masses $\left(m_{\mathrm{e}}^{*}\right.$ and $\left.m_{\mathrm{h}}^{*}\right)$ of $\mathrm{BA}_{2} \mathrm{Pbl}_{4}, \mathrm{EDAPbl}_{4}, \mathrm{DPAPbl}_{4}, \mathrm{BDAPbl}_{4}, \mathrm{PDAPbl}_{4}$, and $\mathrm{HDAPbl}_{4}$. The values in the table are in $m_{0}$, and " $m_{0}$ " is the static mass of a single electron

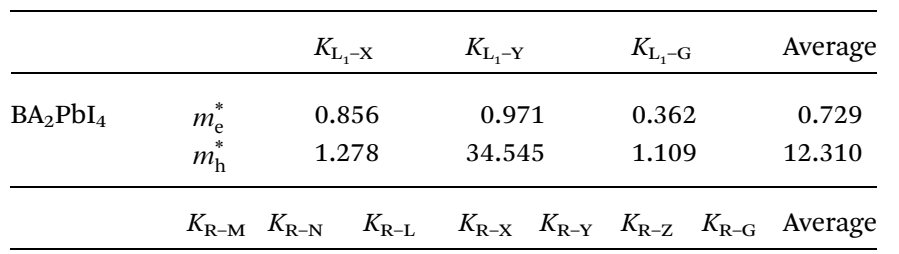

\begin{tabular}{|c|c|c|c|c|c|c|c|c|}
\hline \multirow[t]{2}{*}{$\mathrm{DAPbI}_{4}$} & $\begin{array}{ll}l_{\mathrm{e}}^{*} & 1.079\end{array}$ & 0.935 & 17.122 & 1.200 & 1 & 0 & 0 & \\
\hline & 1.436 & 13.995 & 0.513 & 1.456 & 2.067 & 1.305 & 56 & 3.104 \\
\hline \multirow[t]{2}{*}{$\mathrm{PAPbI}_{4}$} & 1.119 & 0.863 & 2.932 & 1.306 & 1.725 & 0.483 & 0.461 & 1.270 \\
\hline & 4.345 & 2.344 & 0.244 & 0.642 & 0.672 & 2.467 & 1.134 & 1.692 \\
\hline \multirow[t]{2}{*}{$\mathrm{BDAPbI}_{4}$} & $m_{\mathrm{e}}^{*} 0.891$ & 0.741 & 7.368 & 1.036 & 1.231 & 0.228 & 0.266 & 1.680 \\
\hline & $m_{\mathrm{h}}^{*} 0.909$ & 0.480 & 0.465 & 0.842 & 0.610 & 0.660 & 0.535 & 0.643 \\
\hline \multirow[t]{2}{*}{$\mathrm{PDAPbI}_{4}$} & $m_{\mathrm{e}}^{*} \quad 0.887$ & 0.672 & 8.097 & 0.909 & 1.119 & 0.245 & 0.290 & 1.746 \\
\hline & 1.842 & 0.890 & 1.431 & 0.897 & 1.634 & 1.046 & 1.106 & 1.264 \\
\hline \multirow[t]{2}{*}{$\mathrm{HDAPbI}_{4}$} & 0.389 & 0.702 & 20.261 & 0.860 & 0.478 & 0.251 & 0.281 & 3.318 \\
\hline & 0.860 & 0.653 & 63.785 & 0.701 & 1.058 & 0.568 & 0.630 & 9.751 \\
\hline
\end{tabular}

For $2 \mathrm{D}$ perovskite $\mathrm{BA}_{2} \mathrm{PbI}_{4}$, we calculated an effective mass of the $K_{\mathrm{L}_{1}-\mathrm{X}}, K_{\mathrm{L}_{1}-\mathrm{Y}}$, and $K_{\mathrm{L}_{1}-\Gamma}$ directions, as shown in the left side of Fig. 2. The average effective mass of the electrons of the $2 \mathrm{D}$ perovskite $(\mathrm{BA})_{2} \mathrm{PbI}_{4}$ in the three $K$ directions was $0.729 m_{0}$, but the average effective mass of a hole was $12.310 m_{0}$, which is very large. We noted that the effective mass of the electrons demonstrated no obvious anisotropy and was very small in all three $K$ directions, no more than the static mass of electrons $m_{0}$. In contrast, the effective mass of the hole showed significant anisotropy, and the effective mass in the direction of $K_{\mathrm{L}_{1}-\mathrm{Y}}$ was very large, up to $34.545 m_{0}$. From Fig. $1(\mathrm{a})$, we see that the I atom in the direction of base vector a was not equivalent to the I atom in the direction of base vector $b$. The I atom in the direction of $b$ was obviously closer to the amino group of the BA molecule, which results in the obvious distortion of the position of the I atom. The I atom in the direction of vector $a$ was mainly bound to the adjacent $\mathrm{Pb}$ atom and was less affected by the amino group. The band structure also confirmed that the VBM of $\mathrm{BA}_{2} \mathrm{PbI}_{4}$ was generally flatter and less dispersed between $\mathrm{L}_{1}$ and $\mathrm{Y}$.

For the 3D perovskites, starting from point R, we selected the direction parallel to the three basic vectors $\left(K_{\mathrm{R}-\mathrm{M}}, K_{\mathrm{R}-\mathrm{N}}\right.$ and $\left.K_{\mathrm{R}-\mathrm{L}}\right)$, the direction parallel to the facing line $\left(K_{\mathrm{R}-\mathrm{X}}, K_{\mathrm{R}-\mathrm{Y}}\right.$ and $\left.K_{\mathrm{R}-\mathrm{Z}}\right)$, and the direction parallel to the body diagonal $\left(K_{\mathrm{R}-\mathrm{G}}\right)$. According to the calculation results of the average effective mass in the above seven high symmetric directions, it can be seen that the average effective mass values of $\mathrm{DPAPbI}_{4}$, $\mathrm{BDAPbI}_{4}$, and $\mathrm{PDAPbI}_{4}$ were relatively small and no more than $2 m_{0}$. On the other hand, the effective mass of the electron and hole of $\mathrm{EDAPbI}_{4}$ and $\mathrm{HDAPbI}_{4}$ were much larger than $2 m_{0}$. It can be seen that in all of the $3 \mathrm{D}$ perovskites, the effective mass of the electrons in the $K_{\mathrm{R}-\mathrm{M}}$ and $K_{\mathrm{R}-\mathrm{N}}$ directions was significantly smaller than in the $K_{\mathrm{R}-\mathrm{L}}$ direction, which was perpendicular to the $\mathrm{Pb}$ layer and along the base vector $c$. In addition, except for $\mathrm{EDAPbI}_{4}$, as the molecular chain grew longer, the effective mass of electrons in the $K_{\mathrm{R}-\mathrm{L}}$ direction increased significantly from $2.93 m_{0}$ for $\mathrm{DPAPbI}_{4}$ to $20.26 m_{0}$ for $\mathrm{HDAPbI}_{4}$. The reason $\mathrm{EDAPbI}_{4}$ did not satisfy this rule was that two I atoms in the adjacent $\mathrm{Pb}$ atomic layers were too close, and this resulted in mutual exclusion and a large distortion of the atomic arrangement (Fig. S1(a) $\dagger$ ), thus increasing the effective mass in the $K_{\mathrm{R}-\mathrm{L}}$ direction. In this case, the so-called position distortion is that the direction of the connection of the two I atoms has a large angle relative to the $c$ direction. Considering that the electrons at CBM are mainly supplied by the $\mathrm{Pb}$ atoms, with the increased length of the molecular chain, the interlayer 
spacing of the $\mathrm{Pb}$ atoms in the $c$ direction increased from 10.20 $\AA$ for $\mathrm{DPAPbI}_{4}$ to $12.91 \AA$ for $\mathrm{HDAPbI}_{4}$. Thus, the direct migration of electrons between the $\mathrm{Pb}$ atomic layers in this direction is bound to become more and more difficult. By comparing the effective mass of electrons in the different $K$ directions, it was found that the effective mass of electrons in the body diagonal direction $\left(K_{\mathrm{R}-\mathrm{G}}\right)$ was the smallest. In addition, the effective masses in the face diagonal direction $\left(K_{\mathrm{R}-\mathrm{X}}\right.$ and $\left.K_{\mathrm{R}-\mathrm{Y}}\right)$ between the $\mathrm{Pb}$ atoms layers were between that in the body diagonal direction $\left(K_{\mathrm{R}-\mathrm{G}}\right)$ and that in the direction perpendicular to the $\mathrm{Pb}$ atoms layer $\left(K_{\mathrm{R}-\mathrm{L}}\right)$. These results indicated that the electron transport between the layers of the $\mathrm{Pb}$ atoms was easier to achieve by using organic molecules as bridges in the face diagonal and body diagonal directions.

It was found that, except for $\mathrm{HDAPbI}_{4}$, the effective mass of the holes was significantly smaller than the effective mass of the electrons in the $K_{\mathrm{R}-\mathrm{L}}$ direction. Considering that the holes at VBM were mainly provided by the I atoms, the distance between the I atoms in the two adjacent inorganic layers in the $c$ direction was less than $5 \AA$ (Table 1), and the valence wave functions of the I atoms overlap to some extent, which causes the hole carriers to migrate between the I atoms. At the same time, we also observed that with the increase in molecular chain length, the distance between the I atoms in the two adjacent inorganic layers in the $c$ direction increased gradually, which led to the decrease of the overlap of the valence wave functions between the I atoms. As a result, the effective mass of the holes in the $K_{\mathrm{R}-}$ ${ }_{\mathrm{L}}$ direction increased gradually, increasing to $63.785 m_{0}$ in HDA $\mathrm{PbI}_{4}$, which had the largest distance between the I atoms at 6.42 $\AA$. We found that in $\mathrm{EDAPbI}_{4}$ and $\mathrm{DPAPbI}_{4}$, the effective masses of the holes in the inorganic layers ( $K_{\mathrm{R}-\mathrm{M}}$ and $K_{\mathrm{R}-\mathrm{N}}$ directions) were also larger, which may have been due to the larger position distortion of the atoms in the inorganic layers caused by diamino molecules with shorter chains, as shown in Fig. 1(b) and S1(a). $\dagger$ Comparing the effective masses of the electrons and holes in all $K$ directions for the considered perovskites, we found that the effective masses of the electrons and holes for $\mathrm{DPAPbI}_{4}, \mathrm{BDAPbI}_{4}$, and $\mathrm{PDAPbI}_{4}$ were relatively close, although there were differences in the $K_{\mathrm{R}-\mathrm{L}}$ direction. However, the effective masses in most $K$ directions were relatively small and similar to that observed for the monoamine-3D perovskites $\left(\mathrm{MAPbI}_{3}, \mathrm{MASnI}_{3} \text {, and } \mathrm{FAPbI}_{3}\right)^{38,39}$ The carrier mobility is inversely proportional to the effective mass of the carrier. The effective masses of the electrons and holes in the three diamino$3 \mathrm{D}$ perovskites $\left(\mathrm{DPAPbI}_{4}, \mathrm{BDAPbI}_{4}\right.$, and $\left.\mathrm{PDAPbI}_{4}\right)$ were light and balanced, which indicated that their transport properties were excellent. Generally, the interlayer transport properties of mono-amino 2D perovskite materials are poor. For example, 2D perovskite needs to be doped with $\mathrm{TiO}_{2}$ nanowire to improve the interlayer transport properties of the carriers. ${ }^{18}$

\subsection{Optical absorption properties}

It is well known that an excellent candidate for solar energy conversion should have an appropriate bandgap, higher carrier mobility, low carrier recombination rate, as well as a high absorption coefficient. Thus, we simulated optical absorption spectra for all of the $2 \mathrm{D}$ and $3 \mathrm{D}$ perovskites, as shown in Fig. 4 and S4. $\dagger$ The absorption coefficient was calculated using the equation: (a)

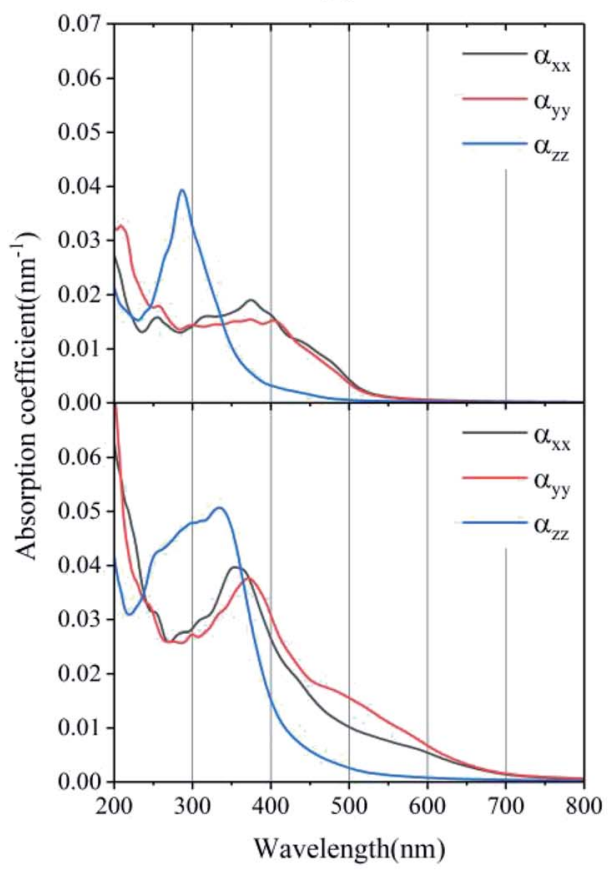

(c) (b)

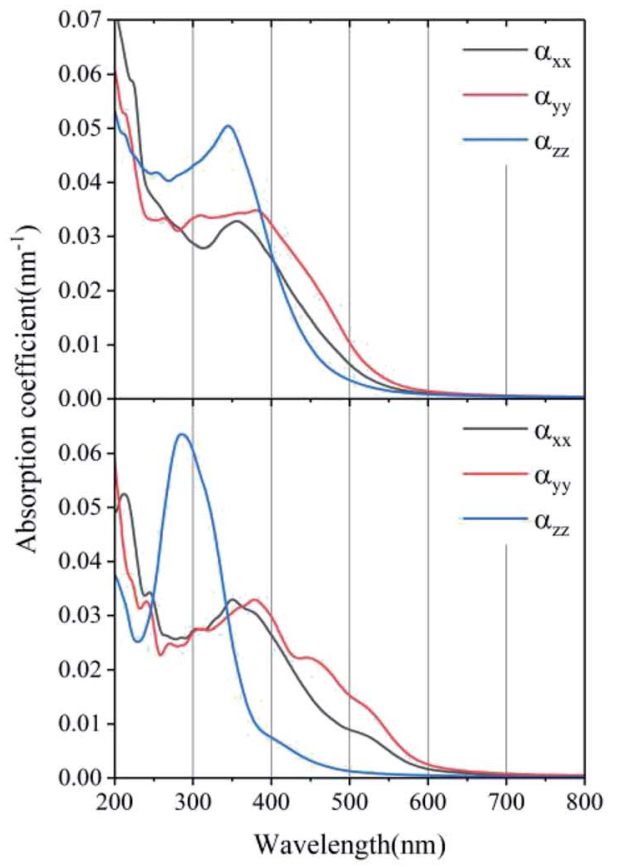

(d)

Fig. 4 Absorption coefficients of (a) $\mathrm{BA}_{2} \mathrm{Pbl}_{4}$, (b) DPAPbl ${ }_{4}$, (c) $\mathrm{BDAPbl}_{4}$, and (d) $\mathrm{PDAPbl}_{4}$. 


$$
I(\omega)=(\sqrt{2}) \omega\left[\sqrt{\varepsilon_{1}(\omega)^{2}+\varepsilon_{2}(\omega)^{2}}-\varepsilon_{1}(\omega)\right]^{\frac{1}{2}}
$$

5where $\varepsilon_{1}(\omega)$ and $\varepsilon_{2}(\omega)$ are the real and imaginary parts of the dielectric function, respectively, depending on the optical frequency $\omega$.

Significant anisotropic optical absorption occurs because of the existence of long-chain monoamino and diamino molecules present in the $2 \mathrm{D}$ and $3 \mathrm{D}$ perovskites. The range of light absorption energy along the direction of the maximum lattice constant $c$ was blue-shifted compared with that in the $\mathrm{Pb}-\mathrm{I}$ inorganic atomic layer, which corresponds to less light absorption in the wavelength range of $400-800 \mathrm{~nm}$, but it contributes greatly to the absorption in the high energy range of less than $400 \mathrm{~nm}$. It was also found that the larger the lattice constant $c$ of the 3D perovskites (except $\mathrm{EDAPbI}_{4}$ ) corresponded to a more obvious blue shift of the optical absorption peak in the $c$ direction. The apparent blue shift of the absorption energy range of $\mathrm{EDAPbI}_{4}$ in the $c$ direction could also be attributed to the position distortion of the two adjacent I atoms between the inorganic layers, as the two I atoms would repel each other. In addition, larger optical absorption coefficients were observed in the wavelength range of $400-800 \mathrm{~nm}$ for all the considered 3D perovskites, which were larger than that of the 2D perovskite $\left(\mathrm{BA}_{2} \mathrm{PbI}_{4}\right)$ in the same wavelength range. It is worth mentioning that the absorption edge of $\mathrm{BDAPbI}_{4}$ had the largest wavelength, which was consistent with the calculated bandgap. For the highest absorption coefficient up to $0.05-0.06 \mathrm{~nm}^{-1}$ for all the calculated 3D perovskites, even in the 550-600 nm wavelength range, the optical absorption coefficients reached $0.01 \mathrm{~nm}^{-1}$, which corresponding to $300 \mathrm{~nm}$-thick perovskites that absorb $90 \%$ of the incident light. The $300 \mathrm{~nm}$ thickness is an optimized value present in several highly efficient planar heterojunction devices. ${ }^{40,41}$ Comparing all of the calculated perovskites, $\mathrm{BDAPbI}_{4}$ exhibit excellent optical absorption properties in the solar light irradiation range, and $\mathrm{BDAPbI}_{4}$ was predicted to exhibit the best photovoltaic performance among all calculated 2D and 3D perovskites, along with a light effective masses of the electrons and holes, a reduced bandgap, and a large optical absorption.

\section{Conclusions}

We studied the geometries, formation energies, electronic structures, and optical absorption properties of $\mathrm{BA}_{2} \mathrm{PbI}_{4}$, $\mathrm{EDAPbI}_{4}, \mathrm{DPAPbI}_{4}, \mathrm{BDAPbI}_{4}, \mathrm{PDAPbI}_{4}$, and $\mathrm{HDAPbI}_{4}$ via firstprinciples calculations. In general, the formation energy of the perovskite materials formed by diamino molecules with longer main chains was relatively low. Although the chain length of the diamino molecule in $\mathrm{DPAPbI}_{4}$ was the same as that of the monoamino molecule in $\mathrm{BA}_{2} \mathrm{PbI}_{4}$, the formation energy of $\mathrm{DPAPbI}_{4}$ was lower than that of $\mathrm{BA}_{2} \mathrm{PbI}_{4}$. We found that the bandgap and band structure depend on the symmetry of the crystal. Among the five 3D perovskite materials, the bandgap of $\mathrm{BDAPbI}_{4}$ was the narrowest direct bandgap at about $1.78 \mathrm{eV}$. All of the 2D and 3D perovskites showed similar results, in that the CBM state was mainly provided by the $\mathrm{Pb}$ atoms, and the VBM state was mainly derived from the I atoms in the $\mathrm{Pb}$ atomic layer. The charge densities in the VBM and CBM states were distinctly separated in space, and a poor overlap of the charge density in the CBM and VBM states could lead to a low recombination transition rate in the diamino-3D perovskites, which is beneficial for obtaining low-loss photovoltaic devices. The transport characteristics of the electrons in the different directions were analyzed by calculating the effective masses in the different directions. The effective masses of the electrons and holes in $\mathrm{DPAPbI}_{4}, \mathrm{BDAPbI}_{4}$, and $\mathrm{PDAPbI}_{4}$ were light and balanced, which indicated that their transport properties were excellent. In particular, $\mathrm{BDAPbI}_{4}$ exhibited excellent optical absorption properties in the solar light irradiation range, along with light effective masses of the electrons and holes, a reduced bandgap, and a large optical absorption. Together, these results will help the future design and synthesis of more efficient and stable organic-inorganic perovskite optoelectronic devices.

\section{Conflicts of interest}

There are no conflicts to declare.

\section{Acknowledgements}

The authors acknowledge the financial support from the National Natural Science Foundation of China (No. 61874008 and 61574014), Beijing Municipal Science and Technology Commission project (No. Z181100004718004), and National Training Program of Innovation and Entrepreneurship for Undergraduates (No. 201910004006).

\section{References}

1 A. Kojima, K. Teshima, Y. Shirai and T. Miyasaka, J. Am. Chem. Soc., 2009, 131, 6050-6051.

2 C. C. Stoumpos, C. D. Malliakas and M. G. Kanatzidis, Inorg. Chem., 2013, 52, 9019-9038.

3 National Renewable Energy Laboratory (NREL) Efficiency chart (2018), http//:www.nrel.gov/pv/assets/pdfs/pvefficiency-chart.20181214.pdf.

4 S. Ahmad, P. K. Kanaujia, H. J. Beeson, A. Abate, F. Deschler, D. Credgington, U. Steiner, G. V. Prakash and J. J. Baumberg, ACS Appl. Mater. Interfaces, 2015, 7, 25227-25236.

5 G. P. Nagabhushana, R. Shivaramaiah and A. Navrotsky, Proc. Natl. Acad. Sci. U. S. A., 2016, 113, 7717-7721.

6 T. A. Berhe, W. N. Su, C. H. Chen, C. J. Pan, J. H. Cheng, H. M. Chen, M. C. Tsai, L. Y. Chen, A. A. Dubale and B. J. Hwang, Energy Environ. Sci., 2016, 9, 323.

7 C. W. Kim, T. Y. Eom, I. S. Yang, B. S. Kim, W. I. Lee, Y. S. Kang and Y. S. Kang, Sci. Rep., 2017, 7, 6849.

8 G. Niu, W. Li, F. Meng, L. Wang, H. Dong and Y. Qiu, J. Mater. Chem. A, 2014, 2, 705.

9 S. Shahbazi, M. Byranvand, F. Tajabadi, S. Afshar and N. Taghavinia, ChemPhysChem, 2016, 17, 2389-2394.

10 G. Niu, X. Guo and L. Wang, J. Mater. Chem. A, 2015, 3, 89708980.

11 J. Schoonman, Chem. Phys. Lett., 2015, 619, 193-195. 
12 I. C. Smith, E. T. Hoke, D. Solis-Ibarra, M. D. McGehee and H. I. Karunadasa, Angew. Chem., Int. Ed., 2014, 126, 1141411417.

13 D. H. Cao, C. C. Stoumpos, O. K. Farha, J. T. Hupp and M. G. Kanatzidis, J. Am. Chem. Soc., 2015, 137, 7843-7850.

14 H. Tsai, W. Nie, J. C. Blancon, C. C. Stoumpos, R. Asadpour, B. Harutyunyan, A. J. Neukirch, R. Verduzco, J. J. Crochet, S. Tretiak, L. Pedesseau, J. Even, M. A. Alam, G. Gupta, J. Lou, P. M. Ajayan, M. J. Bedzyk, M. G. Kanatzidis and A. D. Mohite, Nature, 2016, 536, 312-316.

15 L. N. Quan, M. Yuan, R. Comin, O. Voznyy, E. M. Beauregard, S. Hoogland, A. Buin, A. R. Kirmani, K. Zhao, A. Amassian, D. H. Kim and E. H. Sargent, J. Am. Chem. Soc., 2016, 138, 2649-2655.

16 S. Yang, Y. Wang, P. Liu, Y. B. Cheng, H. J. Zhao and H. G. Yang, Nat. Energy, 2016, 1, 15016.

17 C. C. Stoumpos, D. H. Cao, D. J. Clark, J. Young, J. M. Rondinelli, J. I. Jang, J. T. Hupp and M. G. Kanatzidis, Chem. Mater., 2016, 28, 2852-2867.

18 Y. Sanehira, Y. Numata, M. Ikegami and T. Miyasaka, Chem. Lett., 2017, 46, 1204-1206.

19 H. Tsai, W. Nie, J. C. Blancon, C. C. Stoumpos, R. Asadpour, B. Harutyunyan, A. J. Neukirch, R. Verduzco, J. J. Crochet, S. Tretiak, L. Pedesseau, J. Even, M. A. Alam, G. Gupta, J. Lou, P. M. Ajayan, M. J. Bedzyk, M. G. Kanatzidis and A. D. Mohite, Nature, 2016, 536, 312-316.

20 D. H. Cao, C. C. Stoumpos, O. K. Farha, J. T. Hupp and M. G. Kanatzidis, J. Am. Chem. Soc., 2015, 137, 7843-7850.

21 L. N. Quan, M. Yuan, R. Comin, O. Voznyy, E. M. Beauregard, S. Hoogland, A. Buin, A. R. Kirmani, K. Zhao, A. Amassian, D. H. Kim and E. H. Sargent, J. Am. Chem. Soc., 2016, 138, 2649-2655.

22 G. Kresse and J. Furthmüller, Comput. Mater. Sci., 1996, 6, 15-50.

23 P. E. Blöchl, Phys. Rev. B: Condens. Matter Mater. Phys., 1994, 50, 17953-17979.

24 J. P. Perdew, K. Burke and M. Ernzerhof, Phys. Rev. Lett., 1996, 17, 3865-3868.
25 S. Grimme, J. Antony, S. Ehrlich and S. Krieg, J. Chem. Phys., 2010, 132, 154104.

26 W. Tang, E. Sanville and G. Henkelman, J. Phys.: Condens. Matter, 2009, 21, 084204.

27 E. Sanville, S. D. Kenny, R. Smith and G. Henkelma, J. Comput. Chem., 2007, 28, 899-908.

28 G. Henkelman, A. Arnaldsson and H. Jónsson, Comput. Mater. Sci., 2006, 36, 254-360.

29 E. Mosconi, A. Amat, M. K. Nazeeruddin, M. Gratzel and F. De Angelis, J. Phys. Chem. C, 2013, 117, 13902-13913.

30 J. Even, L. Pedesseau, J.-M. Jancu and C. Katan, J. Phys. Chem. Lett., 2013, 4, 2999-3005.

31 B. Liu, M. Long and M. Cai, Nano Energy, 2019, 715-720.

32 M. M. Lee, J. Teuscher, T. Miyasaka, T. N. Murakami and H. J. Snaith, Science, 2012, 338, 643.

33 (a) D. A. Egger and L. Kronik, J. Phys. Chem. Lett., 2014, 5, 2728-2733; (b) A. Amat, E. Mosconi, E. Ronca, C. Quarti, P. Umari, M. K. Nazeeruddin, M. Gratzel and F. D. Angelis, Nano Lett., 2014, 14, 3608-3616.

34 L. N. Quan, M. Yuan, R. Comin, O. Voznyy, E. M. Beauregard, S. Hoogland, A. Buin, A. R. Kirmani, K. Zhao, A. Amassian, D. H. Kim and E. H. Sargent, J. Am. Chem. Soc., 2016, 138, 2649-2655.

35 D. Li, C. Liang, H. Zhang, C. Zhang, F. You and Z. He, J. Appl. Phys., 2015, 117, 074901.

36 D. Li, J. Meng, Y. Niu, H. Zhao and C. Liang, Chem. Phys. Lett., 2015, 627, 13-19.

37 D. Li, F. Sun, C. Liang and Z. He, J. Alloys Compd., 2018, 741, 489-494.

38 P. Umari, E. Mosconi and F. De Angelis, Sci. Rep., 2014, 4, 4467.

39 A. Amat, E. Mosconi, E. Ronca, C. Quarti, P. Umari, M. K. Nazeeruddin, M. Gratzel and F. De Angelis, Nano Lett., 2014, 14, 3608-3616.

40 D. Liu and T. L. Kelly, Nat. Photonics, 2014, 8, 133.

41 Z. Xiao, C. Bi, Y. Shao, Q. Dong, Q. Wang, Y. Yuan, C. Wang, Y. Gao and J. Huang, Energy Environ. Sci., 2014, 7, 2619-2623. 